\title{
An Initial Analysis of E-Procurement Search Behaviour
}

\author{
Stuart Mackie ${ }^{1}$, Leif Azzopardi ${ }^{2}$, and Yashar Moshfeghi ${ }^{2}$ \\ 1 BiP Solutions Limited, Glasgow, Scotland, UK \\ stuart.mackie@bipsolutions.com \\ 2 University of Strathclyde, Glasgow, Scotland, UK \\ \{leif.azzopardi, yashar.moshfeghi\}@strath.ac.uk
}

\begin{abstract}
Procurement or tender search is where suppliers seek opportunities for providing goods, works or services that authorities, organisations and businesses require. Such opportunities are listed as procurement contract notices for which suppliers can submit tenders. Typically, an E-Procurement system is used to help find and carry out one or more of the stages involved in the procurement process (from finding potential opportunities, bidding on such opportunities, to delivering the goods, works or services, i.e. find, win, deliver). Such systems are crucial in enabling suppliers to efficiently search through the available listings of procurement contract notices listed across various public and commercial portals. However, little research has investigated how end-users search for such opportunities. In this paper, we perform a descriptive analysis of the professional search behaviours of suppliers using a bespoke e-procurement system. Our analysis is based on a sub-sample of six months of search log interaction data. First, we provide an overview of the usage patterns of our sample of users before investigating how the behaviour of searchers is influenced by the type of search form used (quick vs advanced), user expertise (new vs experienced), and the domain of the procurement notices (General, Defence, Medical, etc.). Our findings highlight that more experienced searchers appear to be more strategic than less experienced searchers and that searchers behave differently depending on the domain in terms of querying and assessing behaviours. This analysis suggests that e-procurement search engines need to be mindful of the differences across searchers and between domains when designing a system to help support their users.
\end{abstract}

Keywords: professional search · procurement search.

\section{INTRODUCTION}

Procurement is the activity of tax-funded authorities, organisations and companies purchasing supplies, services, or works. By and large, a majority of procurement opportunities are provided through tax-funded authorities such as national and local government, health, education, police, and defence authorities. Within the European Union (EU), for example, the annual procurement spend accounts 
for approx. $14 \%$ of EU's GDP ${ }^{3}$. Government institutions are increasingly being obliged by law to publish descriptions of public contracts, called, "requests for tenders" or "call for bids". These documents specify the details of the goods, services and/or works required to be supplied and are essentially invitations to suppliers to submit a tender and bid on the contract. Moreover, such public procurement is a highly regulated activity ${ }^{4}$. Public procurement legislation aims to ensure transparency, promote competition, and achieve value for money for taxpayers. Increasingly, commercial organisations are also publishing descriptions of contracts in order to provide greater transparency and increased efficiency. As such, more and more procurement notices are being posted on various and distributed forums (e.g. on local and central government sites, and commercial procurement portals, etc.).

Suppliers that wish to supply to the market can submit bids (i.e. tender) for such procurement contracts. However, prior to a business submitting a tender for a procurement contract, they must first search for and find a relevant opportunity $[6,5]$. The search and recommendation tasks within the procurement contracts search domain can be characterised as a professional search task $[12,10]$ - as the search task has a high financial value associated with it, and the process of searching through all the different opportunities from the disparate platforms comes at a high cost. To this end, various e-procurement systems and search technologies have been developed aimed at helping suppliers identify opportunities for which they can bid on by aggregating the opportunities from the different portals $[8,3,2,9,1]$. Such systems play a vital role in making the market more efficient in matching suppliers with those in need of goods, services and works [7]. Despite their importance, little is known about how suppliers use and engage with such search systems to find relevant opportunities on such bespoke customised search platforms. Such systems are often more complex than web search interfaces [4] and require more expertise to use effectively [13].

In this paper, we present an initial analysis of the search behaviours of professional procurement searchers - people who actively search for opportunities so that their company can submit tenders to win business. Specifically, we present highlights of search user behaviour mined from a large-scale log analysis conducted within the context of a commercial procurement contracts search engine ${ }^{5}$.

To gain a better understanding of search user behaviour within procurement contracts search, we instrumented the search engine and collected user log data over a six-month period [11]. The analysis reported in this paper is based on a 6 -month period from January-June 2020, where we sample all queries and clicks over this time. In Section 2, we first discuss the search context, users, and queries. Then, in Section 3, we analyse customer queries based on the different types of search form used (i.e. advanced vs quick search) and examine the differences between experienced and inexperienced users. Finally, in Section 4, we analyse customer queries across industry domains (i.e. healthcare vs defence).

\footnotetext{
3 ec.europa.eu/growth/single-market/public-procurement_en

${ }_{5}^{4}$ ec.europa.eu/growth/single-market/public-procurement/rules-implementation_en

${ }^{5}$ trackerintelligence.com
} 


\section{Search Context, Users, and Queries}

Several online procurement portals exist for the purpose of publishing procurement notices, such as Contracts Finder ${ }^{6}$, Tenders Electronic Daily ${ }^{7}$, or SAM.gov ${ }^{8}$. Within the market, aggregated search services combine feeds from different country and authority specific portals to reduce the burden on business users of repeatedly searching multiple portals. Within the domain of procurement search, the documents being search for are split into two main categories: contract notices and procurement awards.

Contract notices specify the supplies, services, or works required by the purchasing organisation. Procurement awards disclose which business successfully won a previously advertised contract. Procurement notices are structured documents, with metadata fields denoting the purchaser, location of supply, value of the contract, nature of the contract (i.e. works, services, or supplies), Further, contracts are annotated with the Common Procurement Vocabulary ${ }^{9}$ (CPV), an EU-wide procurement classification system used to assign numerical codes to represent the supplies, services, or works subject to procurement. This metadata is in addition to the contract's full-text natural language description. Within the 6-month period of the log sample we analyse in this paper, we observed that the search engine indexed over 100,000 new contract notices and awards each month. Further, we also noted a marked increase in the volume of contracts published in March 2020, which may correspond to the financial end-of-year or tax-funded authorities publishing procurement tenders related to the COVID-19 pandemic (e.g. for personal protective equipment, etc.).

In contrast to ad-hoc web search [4], we characterise procurement contract search as a professional search task $[12,10]$. This particular search task involves business users (i.e. employees or business owners) searching for economic opportunities, with significant economic value associated with the success of their search tasks. Figure 1 shows the different sizes of the businesses within our sample that submitted queries to the search engine in this period. As can be seen by the distribution over the customer-reported number of employees (captured on sign-up), the search system is used by small, medium, and large businesses - which may impact on the relevance of contract opportunities sought. Additionally, in Figure 1, we illustrate the timestamp of queries and clicks logged. We observe that the temporal pattern of user interaction is directly aligned with traditional business hours (i.e. Monday-Friday 9-5pm) - highlighting that professional searchers are primarily engaged in search activity within the office environment. Taken together, Figure 1 provides us with an initial understanding of the demographics and activities of professional searchers within this domain.

We present summary statistics of search user behaviour within the domain of procurement contracts search in Table 1. In Table 1, we report the mean

\footnotetext{
${ }^{6}$ contractsfinder.service.gov.uk

7 ted.europa.eu/TED

8 beta.sam.gov

${ }^{9}$ simap.ted.europa.eu/web/simap/cpv
} 

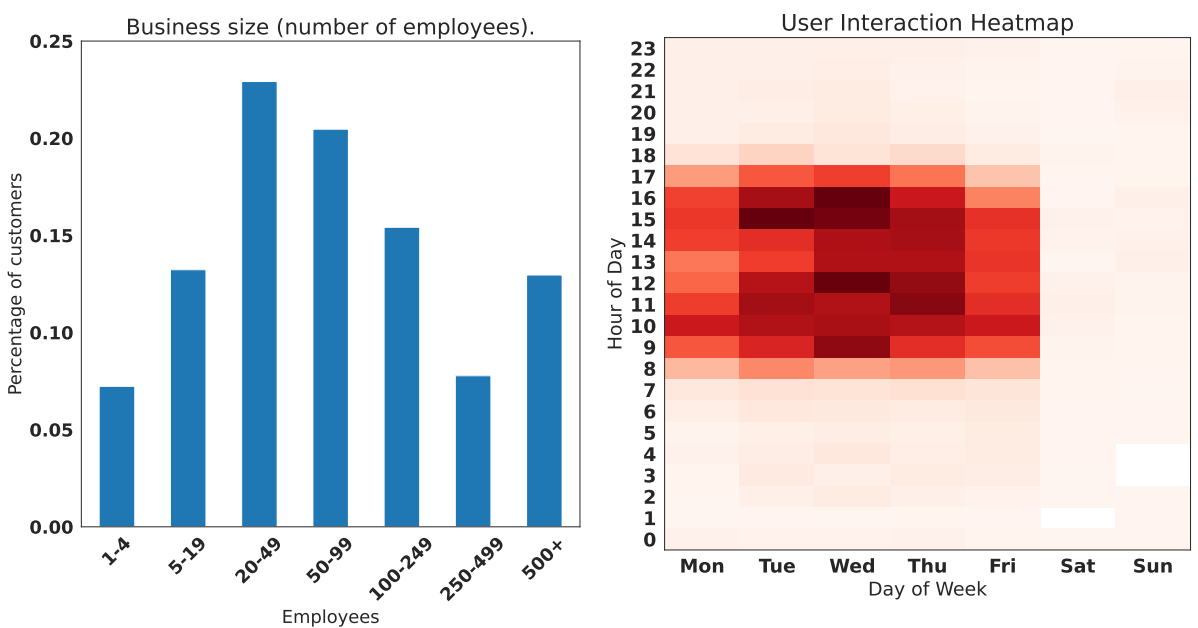

Fig. 1: An illustration of the characteristics of the population of professional searchers within the domain of procurement contracts search. The system is used by customers from a diverse range of business sizes, and is predominately accessed by such users during office hours.

Table 1: Summary statistics of search user behaviour within the domain of procurement contract search.

\begin{tabular}{|c|c|c|c|c|c|}
\hline Terms per-query & Clicks per-query & Click depth & Dwell time & Queries per-session & Session Length \\
\hline 11.2 & 3.0 & 39.3 & $72.3 \mathrm{~s}$ & 1.9 & $8 \mathrm{~m} 49 \mathrm{~s}$ \\
\hline
\end{tabular}

number of terms issued per-query, the mean number of clicks observed perquery, the mean click depth into the SERP rankings, mean dwell time, and then two session-based summary statistics - the number of queries per session and the mean session length. Compared to Web search [4], from Table 1 we note that procurement searchers regularly issue queries with more than ten terms (11.2 vs. 2.35 in Web search), tend to view more pages (3.0 vs. 2.2 in Web search), click on results that are further down the ranking (39.3 vs. half of users not clicking beyond the first Web SERP) and issue more queries in a session (1.9 vs. approx. two-thirds with a single Web search query). We also note the mean dwell time per document examined was approximately one minute while the mean time spend on the search result pages was over eight minutes (across the session), indicating that procurement searchers invest significant amounts of time and effort into searching.

In Figure 2, we illustrate the distribution of total queries over the 6-month log sample, and the distribution of queries observed over different search forms. From Figure 2, we observe that a quarter of all queries within the sample occurred in March 2020, with a sustained increase in search traffic through April, May, and June. This marked increase in the overall number of queries in March 2020 may 

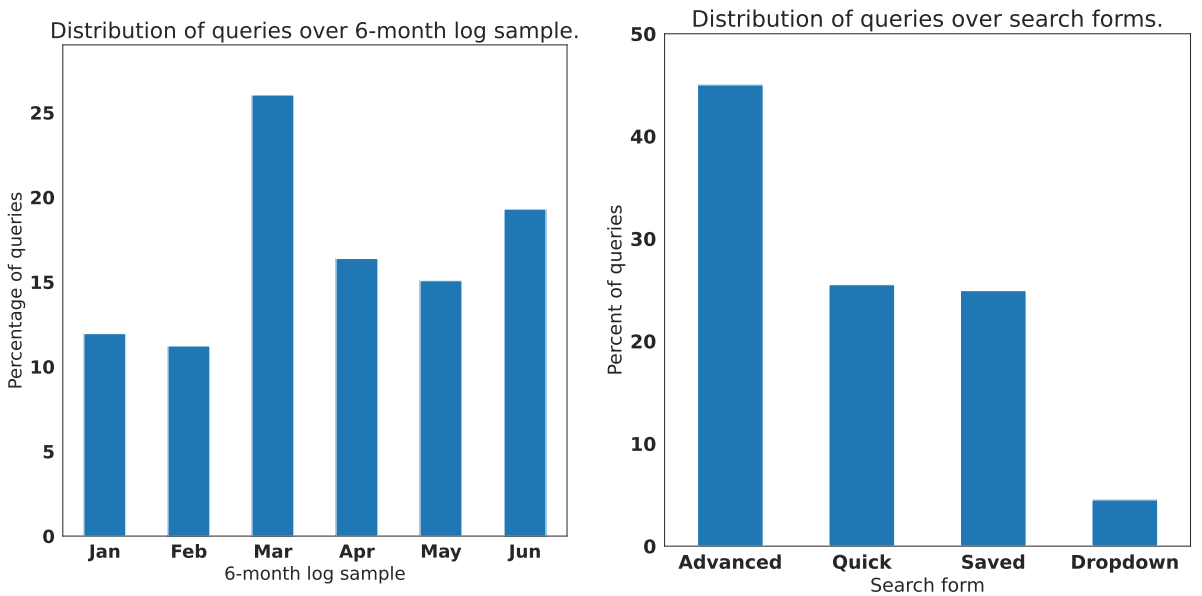

Fig. 2: The distribution of queries over the 6-month log sample, and the distribution of queries over the 4 different search forms offered to users of the system.

Table 2: Distribution of search form usage over the 6-month log sample.

\begin{tabular}{|l|c|c|c|c|c|c|}
\hline Search Form & Jan & Feb & Mar & Apr & May & June \\
\hline Advanced & $51 \%$ & $49 \%$ & $50 \%$ & $41 \%$ & $38 \%$ & $45 \%$ \\
Quick & $25 \%$ & $26 \%$ & $22 \%$ & $26 \%$ & $30 \%$ & $27 \%$ \\
Saved & $21 \%$ & $22 \%$ & $24 \%$ & $28 \%$ & $27 \%$ & $24 \%$ \\
Dropdown & $03 \%$ & $04 \%$ & $04 \%$ & $05 \%$ & $05 \%$ & $04 \%$ \\
\hline
\end{tabular}

potentially be related to the financial end-of-year (with tenders for contracts being related to annual budgets) or the COVID-19 pandemic.

Further from Figure 2, we illustrate the distribution of customer queries submitted to different search forms provided by the system. "Advanced" search is a complex interaction, where the user can specify several search filters when submitting their query, as opposed to "Quick" search where the user simply issues query terms (but can specify an ALL or ANY Boolean condition). "Saved" search allows the user to store a curated query to be executed on the system on a regular basis - and is based on the advanced search form. "Dropdown" search is the simplest form, where the query is expressed as simple query terms with no facility for controlling Boolean operators. From Figure 2, we observe that advanced search is used more frequently than quick search and saved search, with dropdown search accounting for under $5 \%$ of queries submitted to the system. This preference for advanced search further illustrates the investment of time and effort professional searchers are making into this particular search task.

In Table 2, we illustrate the distribution of search form usage over the 6month log sample. From January-March, advanced searches account for approx. half of all queries. Post-COVID19 lockdown (March 2020), we observe a marked change in search behaviour, with a decrease in the monthly proportion of ad- 
Table 3: Search User Behaviour Statistics for New and Experienced Users across the different search type.

\begin{tabular}{|l|c|c|c||c|c|c|}
\hline \multirow{2}{*}{ Interaction } & \multicolumn{3}{|c||}{ New Users } & \multicolumn{3}{c|}{ Experienced Users } \\
\cline { 2 - 7 } & Quick & Advanced & Saved & Quick & Advanced & Saved \\
\hline Query Distribution & 7.8 & 12.5 & 9.5 & 18.0 & $\mathbf{4 0 . 5}$ & 10.0 \\
Query Terms & 2.0 & 8.3 & 21.8 & 1.6 & 10.0 & $\mathbf{3 3 . 5}$ \\
CPV Codes & - & 3.2 & 7.9 & - & 1.5 & $\mathbf{1 3 . 3}$ \\
Query Formulation & $32 \mathrm{~s}$ & $53 \mathrm{~s}$ & $\mathbf{2 m ~ 3 8 s}$ & $36 \mathrm{~s}$ & $1 \mathrm{~m} 28 \mathrm{~s}$ & $1 \mathrm{~m} 39 \mathrm{~s}$ \\
\hline Items Retrieved & 675 & 1920 & 567 & 226 & 707 & 218 \\
Clicks per Query & 2.0 & 2.6 & 3.0 & 1.7 & 2.3 & $\mathbf{3 . 6}$ \\
Rank Click Depth & 33.6 & $\mathbf{6 4 . 5}$ & 64.1 & 15.8 & 31.0 & 38.8 \\
Dwell Time (secs) & 77.93 & 73.93 & 73.97 & 56.18 & $\mathbf{1 1 1 . 2}$ & 76.59 \\
\hline
\end{tabular}

vanced searches, and increases for saved and quick searches. This may be related to employees altering their querying behaviour while working from home and warrants further study in future work.

\section{Search Type Vs. Experience}

In this Section, we investigate procurement searcher's behaviour when using different search forms, and consider what effect experience with the search system may have on this behaviour. Specifically, we analyse log data for "Advanced", "Quick", and "Saved" search form usage for customers who have been subscribed to the system for less than one year (new users), and customers who have been subscribed for more than one year (experienced users). Our research question is: are there observable differences in the characteristics of procurement search behaviour over different search interfaces and user experience levels?

Table 3 reports search user behaviour summary statistics for quick, advanced, and saved search for new users and experienced users. We report the per-form query distribution (i.e. usage), mean number of terms and CPV codes used in queries, the mean time to enter and issue the query (query formulation time), the mean number of items retrieved per-query, the mean number of clicks perquery, the mean of the rank of the last click (rank depth), and the mean of the time spend examining the documents that the user clicked on (dwell time).

In terms of querying behaviour, from Table 3, we first note that $40 \%$ of the total number of queries are for advanced search from experienced users. Over both customer segments, advanced search exhibits the most usage. Beyond advanced search, for new users, there is a moderate preference for saved searches, but for the more experienced users, there is a marked shift towards quick search usage over saved search. Over both customer segments, the number of search terms and CPV codes used in queries increases with the complexity of the search form (i.e. quick $>$ advanced $>$ saved). Specifically, for quick search we see a small number of query terms (with CPV codes being unavailable in quick search), a moderate number of query terms and small number of CPV codes being used for 
advanced search, and a larger number of query terms and increased number of CPV codes for saved searches. Between the two customer segments, we see the new users enter more terms in quick search and more CPV codes in advanced search than the more experienced users. For the more experienced users, we see marked increases in the number of query terms and CPV codes used in curated saved searches. For query formulation time, we see that the time taken to submit queries generally follows the complexity of the search interaction. Advanced search users who have more experience with the system appear to take longer while formulating these searches than novice users, but appear to not spend as much time as novice users curating their saved searches.

In terms of on-SERP behaviour, from Table 3, we note that new users tend to submit queries that return more documents than experienced users. This suggests that experienced users could be obtaining better precision in their results. For clicks per-query, we observe that increases in the number of clicks follows the complexity of the search interaction (i.e. quick $>$ advanced $>$ saved). For click depth, we observe that across each corresponding search form less experienced users tend to click further into the ranking than experienced users, and for both user experience levels the more complex search interactions see increased click depths. The on-document dwell time for new years is similar across search form usage. However, for the more experienced users, there is a marked shift in dwell time between quick search and advanced search. This indicates that more experienced users are being more strategic and are taking less time to assess contracts found via quick search than those found via advanced search - i.e. the amount of time spent assessing is proportional to the amount of time they spend investing in a good query. Table 3 gives us an initial understanding of search user behaviour within the domain of procurement search. From this, we can conclude that there are indeed observable differences in the characteristics of procurement search behaviour over different search interfaces and user experience levels that highlight opportunities for future work.

\section{Query Classification by Industry Sector}

In this Section, we analyse customer queries over different industry domains. The mechanism we use to investigate search user behaviour across industry domains is through categorising queries (and subsequent search interactions) into industry verticals using the CPV codes in queries. CPV codes are added to procurement contracts to indicate the subject of the works, services, or supplied that are to be procured from the market. The categorisation of CPV codes into specific industry domains has been undertaken by procurement experts within the company. Specifically, each CPV code has been mapped to 9 industry verticals: "General", "Defence", "Education \& Culture", "Energy \& Environment", "Health \& Care", "Infrastructure 8 Construction", "Professional Services", "Technology \& Communications", and "Transport \& Logistics". Our research question is: are there are observable differences in the characteristics of procurement search behaviour over different industry groupings? 
Table 4: Search user behaviour statistics - based on industry domain.

\begin{tabular}{|l|c|c|c|c|c|c|}
\hline Industry & Terms & CPV & Q.Form & Clicks & Depth & Dwell \\
\hline General & 22.3 & 28.2 & $1 \mathrm{~m} \mathrm{49s}$ & 5.2 & 33.8 & $60 \mathrm{~s}$ \\
Defence & 25.5 & $\mathbf{5 1 . 0}$ & $1 \mathrm{~m} \mathrm{38s}$ & 6.5 & 41.7 & $42 \mathrm{~s}$ \\
Education \& Culture & 34.3 & 22.0 & $2 \mathrm{~m} \mathrm{3s}$ & 3.7 & 40.9 & $60 \mathrm{~s}$ \\
Energy \& Environment & $\mathbf{6 3 . 3}$ & 25.0 & $1 \mathrm{~m} \mathrm{50s}$ & 6.5 & 37.5 & $40 \mathrm{~s}$ \\
Health \& Care & 29.1 & 24.4 & $1 \mathrm{~m} \mathrm{26s}$ & 5.9 & 56.7 & $\mathbf{7 0 s}$ \\
Infra. \& Construction & 42.5 & 23.6 & $1 \mathrm{~m} \mathrm{34s}$ & 5.6 & 71.9 & $45 \mathrm{~s}$ \\
Professional Services & 44.5 & 20.6 & $1 \mathrm{~m} \mathrm{37s}$ & 5.2 & 47.1 & $47 \mathrm{~s}$ \\
Tech. \& Comms. & 31.1 & 29.1 & $\mathbf{2 m ~ 2 1 s}$ & 4.1 & 38.6 & $53 \mathrm{~s}$ \\
Transport \& Logistics & 29.4 & 36.7 & $2 \mathrm{~m} \mathrm{14s}$ & $\mathbf{6 . 9}$ & $\mathbf{1 0 4 . 7}$ & $62 \mathrm{~s}$ \\
\hline
\end{tabular}

Table 4 reports search user behaviour summary statistics for queries in 9 different industry domains. We report the mean number of terms in queries, the mean number of CPV codes used in queries, the mean query formulation time, the mean number of clicks per-query, the mean rank depth, and mean dwell time. From Table 4, we observe than there is considerable variation in search user behaviour across industry verticals. Specifically, the minimum number of query terms is seen in the "General" category, while the maximum number of query terms is seen in the "Energy $\&$ Environment" category. The "Defence" grouping sees the highest CPV code usage, while the "Professional Services" grouping see the lowest usage of CPV codes in queries. Users who submit queries to the "Education 8 Culture" grouping tend to take the longest to formulate queries. Users who submit queries to the "Transport 83 Logistics" grouping tend to click the most documents per-query and click furthest into the ranking. Users who submit queries to the "Health 8 Care" grouping tend to take the longest to assess contracts. Table 4 gives us a further understanding of search user behaviour within the domain of procurement search. From this, we can conclude that there are indeed observable differences in the characteristics of procurement search behaviour over industry verticals that again highlight opportunities for future work.

\section{Summary and Future Work}

In this paper, via a log study, we have presented an initial overview of search user behaviour of professional searchers within procurement contracts search. We have highlighted interesting differences in demographics and usage that are distinct from general web search, examined the effect that user system experience has on search behaviour, and noted a divergence in behaviour across users within specific industry domains. In doing so, we have ascertained that there are discernible patterns of search user behaviour particular to this professional search task.

The findings presented suggest that specific support is needed for novice searchers so that they can better craft queries and focus their attention when 
searching for contracts. Moreover, our findings also suggest that the different industry domains in which procurement contracts are categorised could also benefit from additional support. However, further work is required to probe more deeply into what mechanism and interventions would best support the searching activities of procurement searchers. Aside from expertise, the notion of relevance also takes on different dimensions: topical and commercial. While traditionally topical relevance is important (i.e. is the document related to type of business opportunities the company is looking?), another important factor is around commercial relevance (i.e. is this opportunity relevant to the business?). While a contract may match a given customer's query and be on topic, a core question is whether the business can actually fulfil the contract, and if they can how profitable or valuable would that contract be to the business? So a small business may not be able to fulfil a large order, while a small order may not worthwhile for a larger businesses. However, such issues around topical relevance and commercial relevance also require further investigation and analysis.

In summary, this work presents one of the first studies providing insights into the search behaviours of users searching for opportunities. And more work is required to better understand the difficulties and challenges in finding and identifying relevant opportunities in order to make the procurement activities more economically efficient, competitive and fair.

\section{Acknowledgments}

The collaboration between BiP Solutions Limited and the University of Strathclyde is supported by Innovate UK (Knowledge Transfer Project 11464).

\section{References}

1. Alvarez-Rodríguez, J., Labra Gayo, J., Silva, F., Alor-Hernández, G., SanchezRamirez, C., Luna, J.: Towards a pan-european e-procurement platform to aggregate, publish and search public procurement notices powered by linked open data: the moldeas approach. International Journal of Software Engineering and Knowledge Engineering (IJSEKE) 22, 365-384 (05 2012)

2. Doring, S., Fischer, S., Kiessling, W., Preisinger, T.: Optimizing the catalog search process for e-procurement platforms. In: International Workshop on Data Engineering Issues in E-Commerce. pp. 39-48 (2005)

3. Döring, S., Kießling, W., Preisinger, T., Fischer, S.: Evaluation and optimization of the catalog search process of e-procurement platforms. Electronic Commerce Research and Applications 5(1), 44-56 (2006), international Workshop on Data Engineering Issues in E-Commerce (DEEC 2005)

4. Jansen, B.J., Spink, A., Bateman, J., Saracevic, T.: Real life information retrieval: A study of user queries on the web. In: ACM SIGIR Forum (1998)

5. Mackie, S., Azzopardi, L., Moshfeghi, Y.: Search User Behaviour within the Procurement Contracts Domain. European Conference on Information Retrieval, Industry Day (2020), ECIR 
6. Mackie, S., Macdonald, D., Azzopardi, L., Moshfeghi, Y.: Looking for Opportunities: Challenges in Procurement Search. In: Proc. of the Conference on Research and Development in Information Retrieval. SIGIR (2019)

7. Mehrbod, A., Zutshi, A., Grilo, A.: A vector space model approach for searching and matching product e-catalogues. In: Proceedings of the Eighth International Conference on Management Science and Engineering Management. pp. 833-842 (2014)

8. Mehrbod, A., Zutshi, A., Grilo, A., Cruz-Machado, V.: Evaluation of an e-catalogue matching mechanism in public procurement notice search 502, 1237-1247 (01 2017)

9. Mynarz, J., Svátek, V., Di Noia, T.: Matchmaking public procurement linked open data. pp. 405-422 (10 2015)

10. Russell-Rose, T., Chamberlain, J., Azzopardi, L.: Information Retrieval in the Workplace: A Comparison of Professional Search Practices. Information Processing \& Management (2018)

11. Silvestri, F.: Mining query logs: Turning search usage data into knowledge. Foundations and Trends in Information Retrieval (2010)

12. Verberne, S., He, J., Kruschwitz, U., Wiggers, G., Larsen, B., Russell-Rose, T., de Vries, A.P.: First International Workshop on Professional Search. SIGIR Forum 52(2) (2018)

13. White, R.W., Dumais, S., Teevan, J.: Characterizing the influence of domain expertise on web search behavior. In: WSDM '09 (2009) 